\title{
Knowledge of the health risks of smoking and impact of cigarette warning labels among tobacco users in six European countries: Findings from the EUREST-PLUS ITC Europe Surveys
}

\author{
Antigona C. Trofor 1,2, Sophia Papadakis ${ }^{3,4}$, Lucia M. Lotrean ${ }^{2,5}$, Cornel Radu-Loghin ${ }^{6}$, Marius Eremia ${ }^{2}$, Florin Mihaltan ${ }^{2,7}$ \\ Pete Driezen ${ }^{8}$, Christina N. Kyriakos ${ }^{3,6}$, Ute Mons $^{9}$, Tibor Demjén ${ }^{10}$, Sarah O. Nogueira ${ }^{11,12}$, Esteve Fernández ${ }^{11,12}$, Yannis \\ Tountas $^{13}$, Krzysztof Przewoźniak ${ }^{14,15}$, Ann McNeill16,17, Geoffrey T. Fong ${ }^{8,18,19}$, Constantine I. Vardavas ${ }^{3,6}$, on behalf of \\ the EUREST-PLUS consortium*
}

\section{ABSTRACT}

INTRODUCTION The aim of this study was to examine knowledge of health effects of smoking and the impact of cigarette package warnings among tobacco users from six European Union (EU) Member States (MS) immediately prior to the introduction of the EU Tobacco Products Directive (TPD) in 2016 and to explore the interrelationship between these two factors.

METHODS Cross-sectional data were collected via face-to-face interviews with adult smokers $(n=6011)$ from six EU MS (Germany, Greece, Hungary, Poland, Romania, Spain) between June-September 2016. Sociodemographic variables and knowledge of health risks of smoking (KHR) were assessed. Warning salience, thoughts of harm, thoughts of quitting and foregoing of cigarettes as a result of health warnings were assessed. The Label Impact Index (LII) was used as a composite measure of warning effects. Linear and logistic regression analyses were used to examine sociodemographic predictors of KHR and LII and the inter-relationship between knowledge and LII scores. RESULTS The KHR index was highest in Romania and Greece and lowest in Hungary and Germany. While the majority of smokers knew that smoking increases the risk for heart diseases, lung and throat cancer, there was lower awareness that tobacco use caused mouth cancer, pulmonary diseases, stroke, and there were very low levels of knowledge that it was also associated with impotence and blindness, in all six countries. Knowledge regarding the health risks of passive smoking was moderate in most countries. The LII was highest in Romania and Poland, followed by Spain and Greece, and lowest in Germany and Hungary. In almost all countries, there was a positive association between LII scores and higher KHR scores after controlling for sociodemographic variables. Several sociodemographic factors were associated with KHR and LII, with differences in these associations documented across countries.

CONCLUSIONS These data provide evidence to support the need for stronger educational efforts and policies that can enhance the effectiveness of health warnings in communicating health risks and promoting quit attempts. Data will serve as a baseline for examining the impact of the TPD.

\section{AFFILIATION}

1 University of Medicine and Pharmacy, 'Grigore T. Popa' lasi, Iasi, Romania

2 Aer Pur România, București, România

3 University of Crete (UoC), Heraklion, Greece

4 Division of Prevention and Rehabilitation, University of Ottawa Heart Institute, Ottawa, Canada

5 Iuliu Hatieganu University of Medicine and Pharmacy, Cluj-Napoca, Romania

6 European Network for Smoking and Tobacco

Prevention (ENSP), Brussels, Belgium

7 University of Medicine and Pharmacy 'Carol Davilla', Bucharest, Romania

8 Department of Psychology, University of Waterloo (UW), Waterloo, Canada

9 Cancer Prevention Unit and WHO Collaborating

Centre for Tobacco Control, German Cancer Research Center (DKFZ), Heidelberg, Germany

10 Smoking or Health Hungarian Foundation (SHHF), Budapest, Hungary

11 Tobacco Control Unit, Catalan Institute of Oncology (ICO), Barcelona, Spain

12 Cancer Control and Prevention Group, Bellvitge Biomedical Research Institute, Barcelona, Spain

13 National and Kapodistrian University of Athens (UoA), Athens, Greece

14 Health Promotion Foundation (HPF), Warsaw, Poland 15 Maria Skłodowska-Curie Institute-Oncology Center (MSCl), Warsaw, Poland

16 National Addiction Centre, Addictions Department, Institute of Psychiatry, Psychology \& Neuroscience, King's College London (KCL), London, United Kingdom 17 UK Centre for Tobacco and Alcohol Studies, London, United Kingdom

18 School of Public Health and Health Systems, University of Waterloo (UW), Waterloo, Canada

19 Ontario Institute for Cancer Research, Toronto, Canada

\section{CORRESPONDENCE TO}

Lucia Maria Lotrean. Iuliu Hatieganu University of Medicine and Pharmacy, Cluj-Napoca, Romania.

E-mail: llotrean@gmail.com

KEYWORDS

tobacco use knowledge, health effects, health warnings, Europe, ITC

Received: 25 July 2018

Revised: 27 October 2018

Accepted: 2 November 2018 


\section{INTRODUCTION}

The World Health Organization Framework Convention on Tobacco Control (WHO FCTC), an unprecedented international treaty aimed at strengthening tobacco control globally, underlines the importance of education, communication, training, and public awareness on the health risks of smoking ${ }^{1}$. A key aspect to educating the public on the deleterious effects of tobacco is the adoption of health warnings on cigarette packages ${ }^{1,2}$. Article 11 of the WHO FCTC addresses packaging and labeling of tobacco products by calling for "each unit packet and package of tobacco products, and any outside packaging and labeling of such products, to carry health warnings describing the harmful effects of tobacco use, and include other appropriate messages' ${ }^{1}$. With the exception of television advertisements, health warnings on cigarette packages are among the most effective sources of information regarding the health effects of smoking, with most smokers reporting becoming more aware about the risks of smoking through warnings on cigarette packages compared to other sources of information ${ }^{2}$. Evidence shows that combined written and graphic health messages are more effective than text-only warnings ${ }^{2-4}$. Pictorial warnings have been shown to increase levels of knowledge regarding the health effects of smoking, as well as increase motivation for smoking cessation behavior among smokers $^{2-9}$.

The updated European Union (EU) Tobacco Products Directives (TPD), which took effect in May 2016 , is a major effort to propel and standardize the ratification of the WHO FCTC among EU Member States (MS), and has reinforced the importance of warning labels as an important tobacco control strategy since they are compelling communication strategy, combining high exposure, high reach, and very low cost ${ }^{10-12}$. In consideration of the strong evidence that health warnings increase awareness of health risks related to tobacco consumption, increase quit attempts and decrease smoking uptake and the realization of the disparities that exist among the EU MS in the implementation of the FCTC, the EU TPD has set forth rules governing the parameters of health warning labels that are scientifically supported to maximize impact. The TPD effectively requires combined text and pictorial health warnings that cover $65 \%$ of the front and back of tobacco product packaging $^{10-12}$.

In many EU countries, full implementation of the 2016 TPD occurred in late fall of 2017. Prior to the TPD coming into effect, EU MS varied in the extent to which they had implemented text and pictorial warnings (Table 1). Moreover, the messages transmitted through health warnings varied across countries (Table 2 ).

In order to evaluate the impact of the TPD provisions on health warnings, it is necessary to establish a better understanding of the knowledge, perceptions, and behaviors related to heath warning labels among EU MS prior to the full implementation of such measures and establishment of a baseline for examining changes over time. This paper reports on knowledge of smoking health harms and the perceived impact of cigarette warning labels among smokers from six EU MS (Germany, Greece, Hungary, Poland, Romania, Spain) before the TPD exerted its full effects, and explores the interrelationship between these two factors as well sociodemographic variables.

\section{Table 1. Description of tobacco product warning labels in countries sampled in $2016^{1}$}

\begin{tabular}{|c|c|c|c|}
\hline Country & Tevt warnings only & $\begin{array}{l}\text { Vedium ( }<50 \% \text { of the front and back } \\
\text { of package) pictorial warnings }\end{array}$ & $\begin{array}{c}\text { Large ( } \geq 50 \% \text { of the front and back of package) } \\
\text { pictorial warnings }\end{array}$ \\
\hline Germany & $\mathrm{x}$ & & Introduced May $2016^{2}$ \\
\hline Greece & $\mathrm{X}$ & & \\
\hline Hungary & & $x$ & Introduced August $2016^{3}$ \\
\hline Poland & $x$ & & Introduced May $2016^{2}$ \\
\hline Romania & & $x$ & \\
\hline Spain & & $x$ & $X^{4}$ \\
\hline
\end{tabular}

1 Information refers to the data collection period (June-September 2016). 2 Introduced May 2016, but slow market penetration due to stockpiling. 3 Introduced on small quantity of packs in market. 4 Even though the TPD was not transposed in Spain at the time of the fieldwork, several manufacturers already sold packages according to the TPD requirements. 
Table 2. Content of health warnings in 2016 by country and Tobaccco Product Directive (TPD) ${ }^{1}$

\begin{tabular}{|c|c|c|c|c|c|c|c|}
\hline Health issuce ${ }^{2}$ & Germany & Greece & Spain & Romania & Itungary & Poland & $T P D^{3,1}$ \\
\hline \multicolumn{8}{|l|}{ Active smoking } \\
\hline Heart disease & Yes & Yes & Yes & Yes & Yes & Yes & Yes \\
\hline Impotence & Yes & Yes & Yes & Yes & Yes & Yes & Yes \\
\hline Lung cancer & Yes & Yes & Yes & Yes & Yes & Yes & Yes \\
\hline Blindness & No & No & No & No & No & No & Yes \\
\hline Mouth cancer & No & No & No & No & No & No & Yes \\
\hline Throat cancer & No & No & Implied ${ }^{5}$ & Implied ${ }^{5}$ & Implied ${ }^{5}$ & No & Yes \\
\hline Stroke & Yes & Yes & Yes & Yes & Yes & Yes & Yes \\
\hline Emphysema & Implied ${ }^{6}$ & Implied ${ }^{7}$ & Implied ${ }^{7}$ & Implied ${ }^{7}$ & Implied ${ }^{7}$ & Implied ${ }^{6}$ & Implied ${ }^{8}$ \\
\hline Bronchitis & Implied ${ }^{6}$ & Implied ${ }^{7}$ & Implied ${ }^{7}$ & Implied ${ }^{7}$ & Implied ${ }^{7}$ & Implied ${ }^{6}$ & Implied ${ }^{8}$ \\
\hline \multicolumn{8}{|l|}{ Passive smoking } \\
\hline Lung cancer & No & Implied ${ }^{9}$ & No & No & No & No & Implied ${ }^{10}$ \\
\hline Heart attacks & No & Implied ${ }^{9}$ & No & No & No & No & Implied ${ }^{10}$ \\
\hline Asthma in children & Implied ${ }^{11}$ & Implied ${ }^{11}$ & Implied ${ }^{12}$ & Implied ${ }^{12}$ & Implied ${ }^{12}$ & Implied ${ }^{12}$ & Implied ${ }^{10}$ \\
\hline
\end{tabular}

\section{METHODS}

\section{Study sample and methodology for data collection}

The current study is part of a European Commission Horizon-2020 funded study entitled European Regulatory Science on Tobacco: Policy implementation to reduce lung diseases (EUREST-PLUSHCO-06-2015). The EUREST-PLUS Project aims to monitor and evaluate the impact of the implementing Acts of the TPD within the context of the FCTC and its implementation within Europe ${ }^{13}$. Using the methodology of The International Tobacco Control Policy Evaluation (ITC) Project, a cross-sectional survey was conducted among six European countries (Wave $16 \mathrm{E})^{14}$. A nationally representative sample of 6011 cigarette smokers of age 18 years or older was collected from Germany ( $\mathrm{n}=1003)$, Greece $(\mathrm{n}=1000)$, Hungary $(n=1000)$, Poland $(n=1006)$, Romania $(n=1001)$, and Spain $(n=1001)$. Ethics approval for data collection was provided for each national cohort by the corresponding local ethics committees and informed consent was received from all participants.

Data collection occurred between June 2016 and September 2016. According to Nomenclature of Territorial Units for Statistics (NUTS), the geographic strata were NUTS2 regions (NUTS1 in Germany) crossed with degree of urbanization (urban, semi- urban, rural). Approximately 100 area clusters were sampled in each country, with the aim of obtaining 10 adult smokers per cluster. Clusters were allocated to strata proportionally to the population size for adults 18 years and older. Within each cluster, household addresses were sampled using a random walk design. One randomly selected male smoker and one randomly selected female smoker were chosen for interview from a sampled household where possible. Screening of households continued until the required number of smokers from the cluster had been interviewed. All interviews were conducted face-to-face by interviewers using tablets. Further details on the methodology of the $6 \mathrm{E}$ Survey can be found elsewhere ${ }^{13}$.

During the data collection period (June-Sept. 2016), Greece had text warnings on tobacco packages, while Germany and Poland had just moved from text warnings to large pictorial warnings covering $\geq 50 \%$ of the front and back of the package in May 2016. Romania, Hungary and Spain had mediumsize pictorial warning $(<50 \%$ of the front and back of package) which had been in place since 2008 (Romania) or 2011 (Hungary and Spain); in Hungary since 20 August 2016, there may have been tobacco products with large pictorial warnings, but compared to all, probably only to a negligible extent (Table 1). 


\section{Measures}

Knowledge of health risks

In order to assess knowledge of the health risks of smoking, respondents were presented with nine diseases and conditions (heart disease, impotence, lung cancer, blindness, mouth cancer, throat cancer, stroke, emphysema, bronchitis) and were asked to state whether they believed the condition is caused by smoking. Respondents were also asked to state if they believed secondhand smoke caused lung cancer in non-smokers, heart attacks in non-smokers, and asthma in children. Knowledge of health risks was coded as $0=$ 'not caused by smoking/don't know' and $1=$ 'caused by smoking'. An index regarding knowledge of the health risks of smoking (KHR) was calculated by summing the standardized scores (z-scores calculated across all countries) of each participant's responses to the questions pertaining to health risks of smoking.

\section{Warning label impact}

Warning label effects were measured using six indicators as in previous ITG surveys ${ }^{7,8,15-16}$. Each indicator was analyzed as a dichotomous measure of frequency. Warning label salience was assessed using two questions: 1) NOTICING: 'in the last month, how often, if at all, have you noticed the warning labels on cigarette packages $(1=$ very often or often; $0=$ sometimes, rarely, or never)?', and 2) READING: 'In the last month, how often, if at all, have you read or looked closely at the warning labels on cigarette packages $(1=$ often; $0=$ never or once in a while)?'. THOUGHTS of harm were assessed with the following question: 'to what extent, if at all, do the warning labels make you think about the health risks of smoking ( $1=\mathrm{a}$ lot; $0=$ somewhat, a little, or not at all)?'. Thoughts of QUITTING were assessed with the question: 'to what extent, if at all, do the warning labels on cigarette packs make you more likely to quit smoking ( $1=\mathrm{a}$ lot; $0=$ somewhat, a little, or not at all)?'. FORGOING of cigarettes was assessed by asking: 'in the last month, have the warning labels stopped you from having a cigarette when you were about to smoke one ( $1=$ many times, a few times, or once; $0=$ never $)$ ?'. AVOIDING was assessed: 'in the last month, have you made any effort to avoid looking or thinking about the warning labels, such as covering them up, keeping them out of sight, using a cigarette case, avoiding certain warnings, or any other means ( $1=$ yes, $0=$ no $)$ ?'.

The Labels Impact Index (LII) was calculated using the methodology employed in several previous ITC reports ${ }^{7,8,15-17}$. The LII is a composite measure that combines four of the six warning label effectiveness indicators and then weighting and summing the standardized scores (z scores) using the following calculation: $\mathrm{LII}=($ NOTICING $\times 1)+($ THOUGHTS $\times 2)+($ QUITTING $\times 2)+($ FORGOING $\times 3)$. The LII was calculated using the original four/five-point scales of the individual measures of health warning effectiveness, not dichotomized. Higher LII scores indicate greater impact ${ }^{16}$.

Respondents were also asked if they would like to have more, less or the same amount of information contained in the health warnings.

\section{Demographics and smoking behavior}

Sociodemographic characteristics including country, age, gender, degree of urbanization (urban, semiurban, rural), level of education (low, medium, high) and income level (low, medium, high) were assessed. Highest level of formal education completed categorized as: low (primary, lower pre-vocational secondary, middle pre-vocational secondary), moderate (secondary vocational, senior general secondary and pre-university) and high (higher professional and university Bachelor, university Masters). Due to the different income cut-off points across countries, income was categorized as low, medium, and high using standardized country-level cut-offs established by the ITC Project ${ }^{18}$ as follows: low income level $(<€ 1750$ for Germany, Greece and Spain; $\leq 150000$ Ft for Hungary; $\leq 2000$ zł for Poland; $\leq 1000$ lei for Romania), moderate income ( $€ 1750$ to $€ 3000 ; 150001 \mathrm{Ft}$ to $250000 \mathrm{Ft} ; 2001 \mathrm{zt}$ to 4000 zł; 1001 lei to 2500 lei), and high income (>€3000, $>250000 \mathrm{Ft},>4000 \mathrm{zl},>2500$ lei). Smoking behavior included the assessment of number of cigarettes smoked per day $(<10,11-20,21-30,>30)$.

\section{Statistical analyses}

Data were analyzed with SPSS 20.0 using the Complex Samples package to account for the complex sampling design. We analyzed data for each country separately and calculated percentages and standard errors. All statistical estimates presented are weighted for sex, 
age and degree of urbanization to make the data more representative of the population of smokers in each country. Participants who did not offer information regarding sociodemographic characteristics or the variables assessing the knowledge about health risks of smoking or the warning label impact were excluded from the analyses. Linear regression analyses were conducted to examine predictors of KHR and LII, the independent variables being age, gender, degree of urbanization, income, and education level, as well as the number of cigarettes smoked per day; these approaches were used based on previous relationships tested in other ITC studies ${ }^{4,8,15}$.

In order to assess if the label impact index influences knowledge regarding the health risks of smoking, two approaches were used. First, linear regressions were performed with the KHR index as the dependent variable and the LII index as the independent variable, controlling for sociodemographic variables, as well as the number of cigarettes smoked per day. Second, logistic regression was performed using as dependent variables the measures that assess the knowledge of the risk of smoking for several diseases, and using the LII index as the independent variable, controlling for age, gender, degree of urbanization, income, education level (sociodemographic variables) as well as the number of cigarettes smoked per day.

\section{RESULTS}

\section{Characteristics of the study sample}

The study sample consisted of 6011 persons $(47.2 \%$ women and $52.8 \%$ men; $35.4 \%$ were from urban areas, $37.5 \%$ from semi-urban and $27.1 \%$ from rural areas). The mean age was 45.1 years $(8.4 \%, 18-24$ years; $29.5 \%, 25-39 ; 33.3 \%, 40-54 ; 28.7 \%$ older than 54 years). The education level was: $36.8 \%$ low, $51.6 \%$ medium, $11 \%$ high, $0.6 \%$ did not declare. With regard to the income, $22.2 \%$ had low income, $37.4 \%$ medium, $17.4 \%$ high income, while $23 \%$ did not declare.

\section{Knowledge of health risks (KHR) of smoking}

With the exception of Hungary, more than $80 \%$ of the smokers in each country knew that active smoking causes heart diseases, lung cancer, and throat cancer (Table 3). More than two-thirds of smokers from all countries were aware of the risk of active smoking for pulmonary diseases (emphysema, bronchitis) and mouth cancer. In Spain and Hungary less than $60 \%$ of the smokers were aware of the risk of active smoking for stroke. The effect of smoking on impotence was recognized by approximately half of smokers and on blindness by one-third or less, the only exception being Romania where almost two-thirds knew the risk of smoking for impotence and more than half knew that smoking can cause blindness. Knowledge

Table 3. Knowledge of the health risks (KHR) of smoking overall and by country ( $n=6011)$

\begin{tabular}{|c|c|c|c|c|c|c|c|}
\hline \multirow[b]{2}{*}{ Ilealth Ellect } & Overall & Germany & Greece & Spain & Romania & Hungary & Poland \\
\hline & \multicolumn{7}{|c|}{ Per cent who agreed (SE) } \\
\hline \multicolumn{8}{|l|}{ Active smoking } \\
\hline Heart disease & $82.7(1.0)$ & $81.9(2.8)$ & $94.9(1.1)$ & $81.9(2.5)$ & $87.7(1.6)$ & $67.0(3.1)$ & $82.7(2.6)$ \\
\hline Impotence & $56.1(1.3)$ & $50.2(3.5)$ & $61.7(3.0)$ & $53.7(2.9)$ & $64.3(2.6)$ & $54.7(3.3)$ & $52.0(3.3)$ \\
\hline Lung cancer & $90.0(0.7)$ & $89.8(2.6)$ & $96.1(0.8)$ & $93.1(1.8)$ & $90.2(1.2)$ & $83.4(1.9)$ & $87.4(1.9)$ \\
\hline Blindness & $34.7(1.2)$ & $23.8(2.6)$ & $33.0(3.1)$ & $24.4(3.2)$ & $57.6(2.8)$ & $35.7(3.3)$ & $33.9(2.8)$ \\
\hline Mouth cancer & $78.0(0.9)$ & $71.3(3.0)$ & $85.3(1.6)$ & $84.7(2.0)$ & $76.7(2.0)$ & $70.7(2.6)$ & $79.3(2.4)$ \\
\hline Throat cancer & $83.1(0.9)$ & $82.3(2.8)$ & $88.0(1.4)$ & $78.5(2.3)$ & $88.6(1.3)$ & $76.7(2.3)$ & $84.8(2.2)$ \\
\hline Stroke & $65.2(1.1)$ & $71.5(3.2)$ & $68.6(2.6)$ & $56.4(2.9)$ & $78.1(1.8)$ & $55.6(3.0)$ & $61.0(2.9)$ \\
\hline Emphysema & $72.5(1.1)$ & $68.7(2.9)$ & $78.6(2.1)$ & $75.2(2.5)$ & $66.2(3.1)$ & $69.1(2.8)$ & $77.2(2.8)$ \\
\hline Bronchitis & $79.5(1.0)$ & $77.4(2.7)$ & $88.4(1.7)$ & $92.2(1.7)$ & $80.8(1.7)$ & $68.2(3.0)$ & $69.7(3.0)$ \\
\hline \multicolumn{8}{|l|}{ Secondhand smoke } \\
\hline Lung cancer & $68.2(1.2)$ & $58.4(3.4)$ & $70.1(3.1)$ & $67.3(3.6)$ & $82.3(1.7)$ & $57.9(3.2)$ & $72.8(2.7)$ \\
\hline Heart attacks & $56.3(1.3)$ & $41.1(3.4)$ & $63.2(3.2)$ & $51.3(3.7)$ & $73.4(2.4)$ & $43.5(3.5)$ & $65.1(3.0)$ \\
\hline \multirow[t]{2}{*}{ Asthma in children } & $68.5(1.2)$ & $59.8(3.6)$ & $73.8(2.8)$ & $68.2(2.7)$ & $78.9(2.0)$ & $63.9(3.2)$ & $66.5(2.8)$ \\
\hline & \multicolumn{7}{|c|}{ Vean (SE) } \\
\hline KHR Index ${ }^{a}$ & $-0.046(0.220)$ & $-1.154(0.662)$ & $1.614(0.371)$ & $-0.110(0.514)$ & $1.868(0.435)$ & $-2.029(0.610)$ & $0.002(0.583)$ \\
\hline
\end{tabular}

SE: standard error. a KHR Index, calculated using z scores. 
regarding the health risks of passive smoking was moderate in most countries, with lower levels of knowledge documented in Germany and Hungary. The risks of passive smoking were best known by smokers in Romania (Table 3). Smokers from Hungary and Germany also reported the lowest KHR index score, while the highest scores were documented in
Greece and Romania.

The results of the regression analysis examining predictors or KHR are reported in Table 4. KHR index scores were lower among men than women in Germany, Hungary and Poland. In Romania and Greece, significantly lower KHR index scores were documented for smokers of age 18-24 years,

Table 4. Predictors of knowledge of the health risks (KHR) of smoking index (results of linear regression analysis)

\begin{tabular}{|c|c|c|c|c|c|c|}
\hline \multirow[b]{2}{*}{ Variable } & Germany & Greece & Spain & Romania & Ilungary & Poland \\
\hline & \multicolumn{6}{|c|}{ B $\left(95^{\circ} \%\right.$ CI $)$} \\
\hline \multicolumn{7}{|l|}{ Age group } \\
\hline \multirow{2}{*}{$18-24$} & 0.45 & $-2.72^{*}$ & 0.53 & $-3.29^{* *}$ & -2.63 & 0.07 \\
\hline & $(-2.19,3.08)$ & $(-5.24,-0.20)$ & $(-3.05,4.12)$ & $(-5.35,-1.22)$ & $(-7.16,1.90)$ & $(-2.76,2.90)$ \\
\hline \multirow[t]{2}{*}{ 25-39 } & 0.57 & -0.01 & -0.205 & $-2.02^{* *}$ & -1.08 & -1.12 \\
\hline & $(-1.60,2.74)$ & $(-1.03,1.01)$ & $(-2.61,2.20)$ & $(-3.45,-0.58)$ & $(-3.72,1.55)$ & $(-3.24,0.99)$ \\
\hline \multirow[t]{2}{*}{$40-54$} & 1.37 & 0.06 & 0.88 & 0.59 & -0.27 & 0.58 \\
\hline & $(-0.68,3.42)$ & $(-1.15,1.27)$ & $(-0.39,2.15)$ & $(-0.68,1.88)$ & $(-2.46,1.92)$ & $(-1.11,2.27)$ \\
\hline$>54$ & Ref & Ref & Ref & Ref & Ref & Ref \\
\hline \multicolumn{7}{|l|}{ Gender } \\
\hline \multirow[t]{2}{*}{ Male } & $-1.59^{* * *}$ & -0.49 & 0.19 & -0.06 & $-1.64^{*}$ & $-1.30^{*}$ \\
\hline & $(-2.50,-0.68)$ & $(-1.46,0.48)$ & $(-1.49,1.90)$ & $(-1.56,1.45)$ & $(-3.01,-0.28)$ & $(-2.38,-0.22)$ \\
\hline Female & Ref & Ref & Ref & Ref & Ref & Ref \\
\hline \multicolumn{7}{|c|}{ Urbanization degree } \\
\hline \multirow[t]{2}{*}{ Urban } & 0.50 & 0.19 & -0.23 & 0.63 & 1.60 & 0.47 \\
\hline & $(-2.92,3.93)$ & $(-2.12,2.51)$ & $(-2.45,2.40)$ & $(-1.39,2.66)$ & $(-2.58,5.79)$ & $(-2.30,3.24)$ \\
\hline \multirow[t]{2}{*}{ Semi-urban } & 1.23 & 1.28 & 0.40 & 1.30 & 2.66 & -1.280 \\
\hline & $(-1.30,3.95)$ & $(-0.63,3.19)$ & $(-2.13-2.93)$ & $(-1.10,3.70)$ & $(-1.40,6.73)$ & $(-4.04,1.48)$ \\
\hline Rural & Ref & Ref & Ref & Ref & Ref & Ref \\
\hline \multicolumn{7}{|l|}{ Education } \\
\hline \multirow[t]{2}{*}{ Low } & -2.18 & -0.96 & -1.60 & -0.65 & 1.09 & -1.32 \\
\hline & $(-5.1,0.64)$ & $(-2.52,0.59)$ & $(-3.70,0.52)$ & $(-3.56,2.25)$ & $(-3.43,5.62)$ & $(-4.21,1.57)$ \\
\hline \multirow[t]{2}{*}{ Medium } & -0.78 & 0.57 & -1.38 & -0.72 & 0.46 & -0.258 \\
\hline & $(-2.94,1.38)$ & $(-0.72,1.86)$ & $(-3.28,0.52)$ & $(-2.43,0.98)$ & $(-4.07,4.99)$ & $(-2.44,1.93)$ \\
\hline High & Ref & Ref & Ref & Ref & Ref & Ref \\
\hline \multicolumn{7}{|l|}{ Income } \\
\hline \multirow[t]{2}{*}{ Low } & -1.10 & 2.33 & -1.72 & 0.36 & -1.55 & -0.35 \\
\hline & $(-3.49,1.29)$ & $(-0.96,4.75)$ & $(-3.80,0.36)$ & $(-2.53,3.25)$ & $(-4.21,1.11)$ & $(-2.50,1.81)$ \\
\hline \multirow[t]{2}{*}{ Medium } & -0.85 & 1.77 & 1.52 & 1.04 & -1.00 & -1.12 \\
\hline & $(-2.43,0.74)$ & $(-0.32,3.87)$ & $(-0.78,3.84)$ & $(-0.86,3.00)$ & $(-3.02,1.02)$ & $(-3.08,0.62)$ \\
\hline High & Ref & Ref & Ref & Ref & Ref & Ref \\
\hline \multicolumn{7}{|c|}{ Cigarettes/day } \\
\hline \multirow[t]{2}{*}{$1-10$} & 1.14 & 0.67 & 0.20 & 4.62 & 2.66 & 2.02 \\
\hline & $(-4.03,6.17)$ & $(-1.09,2.44)$ & $(-2.88,3.29)$ & $(-1.08,10.32)$ & $(-3.44,8.76)$ & $(-1.96,6.01)$ \\
\hline \multirow[t]{2}{*}{$11-20$} & -0.65 & 0.37 & -0.09 & 3.32 & 1.93 & 2.68 \\
\hline & $(-4.05,6.34)$ & $(-1.20,1.94)$ & $(-3.01,2.84)$ & $(-2.50,9.14)$ & $(-4.19,8.05)$ & $(-1.20,6.56)$ \\
\hline \multirow[t]{2}{*}{$21-30$} & 0.98 & -0.73 & -0.58 & 3.60 & 2.73 & $3.86^{*}$ \\
\hline & $(-4.26,6.24)$ & $(-2.67,1.20)$ & $(-4.01,2.84)$ & $(-2.27,9.48)$ & $(-4.17,9.65)$ & $(0.22,7.71)$ \\
\hline$>30$ & Ref & Ref & Ref & Ref & Ref & Ref \\
\hline
\end{tabular}

Missing cases were excluded from the analysis. Ref: indicates the reference group in the linear regression analyses. ${ }^{*} p<0.05,{ }^{* *} p<0.01,{ }^{* * *} p<0.001$. 
compared to older age groups. In Poland, smokers who smoke less cigarettes/day had a better score. No other significant associations were documented for income or education at the country-level.

\section{Effects of warning labels}

The proportion of smokers who reported noticing warning labels 'often or very often' varied across countries (Table 5). Specifically, rates were lowest in Greece and Hungary, and highest in Romania and Poland where more than half of respondents reported they noticed the warnings. Less than one in five smokers declared that they read the warning labels often or very often, except in Romania where onethird of the smokers declared this.

The percentage of smokers who recalled seeing a helpline number on cigarette packs was around $30 \%$ in Germany, Romania and Poland, but was lower than $22 \%$ in Hungary and Spain, while in Greece it was around $7 \%$. Information regarding quitting websites was noticed on cigarette packs by about $15 \%$ of smokers in all countries, ranging from the highest in Germany (20.9\%) to the lowest in Greece $(5.4 \%)$.

The percentage of smokers who declared that the warning labels made them think 'a lot' about health risks or made them more likely to quit was less than $6 \%$ for all countries, except in Romania, where more than 10\% declared this. In Germany, Greece, Spain and Romania, 15\% or less of smokers declared that in the last 30 days warning labels made them refrain from smoking at least once, while the percentage in Hungary and Poland was around $20 \%$.

LII scores were lowest in Germany and Hungary, followed by Greece and Spain, and highest in Poland and Romania (Table 5). In all countries, except for Spain, the LII was higher among respondents who reported lower daily cigarette consumption (Table 6). In Poland, the LII was lower among people with lower educational level, while in Romania it was higher among those with low income. In Poland and Romania, it was lower among younger age groups, while in Greece a reverse situation was found. In Germany, urban residence was positively associated with a higher LII, while in Poland, the LII was lower among men.

Around two-thirds of smokers reported they wanted the same amount of health information to appear on cigarette packages, with the exception of Germany where more than $40 \%$ of smokers reported wanting less information on warning labels.

Table 5. Effects of warning labels and Label Impact Index (LII) scores overall and by country (n=6011)

\begin{tabular}{|c|c|c|c|c|c|c|c|}
\hline & Overall & Germany & Grecee & Spain & Romania & Itungary & Poland \\
\hline Variable & $\%(\mathrm{SE})$ & $\%($ SE) & $\%(\mathrm{SE})$ & $\%(\mathrm{SE})$ & $\%(S E)$ & $\%(\mathrm{SE})$ & $\%($ SE) \\
\hline Noticing & $38.3(1.1)$ & $30.7(2.7)$ & $21.4(2.4)$ & $36.6(3.0)$ & $56.5(2.8)$ & $25.2(2.6)$ & $59.2(2.9)$ \\
\hline Reading & $20.2(0.9)$ & $11.5(1.5)$ & $21.9(2.5)$ & $12.6(1.2)$ & $37.6(2.9)$ & $15.0(2.3)$ & $20.7(2.4)$ \\
\hline Thoughts & $5.6(0.4)$ & $4.7(1.2)$ & $5.1(1.0)$ & $5.1(1.0)$ & $12.8(1.3)$ & $3.0(0.8)$ & $3.3(0.9)$ \\
\hline Quitting & $4.0(0.4)$ & $3.2(0.9)$ & $2.5(0.7)$ & $2.4(0.6)$ & $10.6(1.3)$ & $1.4(0.5)$ & $3.9(1.0)$ \\
\hline Foregoing & $15.4(0.8)$ & $10.3(1.5)$ & $13.5(1.7)$ & $14.2(2.2)$ & $14.5(1.7)$ & $18.3(2.3)$ & $21.6(2.6)$ \\
\hline Avoidance & $13.3(0.7)$ & $9.9(1.4)$ & $14.2(1.6)$ & $19.9(1.9)$ & $16.5(2.0)$ & $9.2(1.8)$ & $10.4(1.6)$ \\
\hline \multicolumn{8}{|l|}{ Saw quitting supports } \\
\hline Helpline number ${ }^{b}$ & $24.0(1.0)$ & $28.6(2.3)$ & $6.5(1.3)$ & $19.2(2.4)$ & $36.3(2.6)$ & $21.8(3.0)$ & $31.4(2.4)$ \\
\hline Quitting website & $15.2(0.8)$ & $20.9(2.1)$ & $5.4(1.1)$ & $15.7(1.7)$ & $17.9(1.9)$ & $14.8(2.3)$ & $16.4(1.9)$ \\
\hline \multicolumn{8}{|l|}{ Health Information } \\
\hline Want more & $14.4(0.7)$ & $8.4(1.3)$ & $23.5(2.1)$ & $14.9(1.7)$ & $25.1(2.2)$ & $3.0(0.6)$ & $11.9(1.3)$ \\
\hline Want the same & $63.1(1.1)$ & $46.5(3.0)$ & $63.8(2.7)$ & $61.2(2.3)$ & $60.6(2.7)$ & $72.2(2.7)$ & $74.3(2.1)$ \\
\hline \multirow[t]{2}{*}{ Want less } & $18.3(0.9)$ & $40.0(3.0)$ & $10.9(1.7)$ & $18.8(2.0)$ & $11.1(1.3)$ & $21.9(2.5)$ & $7.3(1.4)$ \\
\hline & \multicolumn{7}{|c|}{ Mean Score (SE) } \\
\hline Label Impact Index (LII) & $-0.071(0.147)$ & $-1.090(0.327)$ & $-0.335(0.364)$ & $-0.378(0.318)$ & $1.519(0.343)$ & $-0.650(0.378)$ & $0.507(0.422)$ \\
\hline
\end{tabular}


Table 6. Predictors of the Label Impact Index (LII) (results of linear regression analysis)

\begin{tabular}{|c|c|c|c|c|c|c|}
\hline \multirow[b]{2}{*}{ Variable } & Germany & Greece & Spain & Romania & Ilungary & Poland \\
\hline & \multicolumn{6}{|c|}{$B\left(95^{\circ} \% \mathrm{CI}\right)$} \\
\hline \multicolumn{7}{|l|}{ Age group } \\
\hline \multirow[t]{2}{*}{$18-24$} & 1.11 & -0.20 & 1.01 & $-2.27^{* *}$ & -1.08 & 0.07 \\
\hline & $(-0.29,2.52)$ & $(-1.88,1.49)$ & $(-1.44,3.45)$ & $(-3.69,-0.84)$ & $(-3.45,1.28)$ & $(-2.28,2.42)$ \\
\hline \multirow[t]{2}{*}{$25-39$} & 0.50 & $1.52^{*}$ & -1.09 & -0.82 & 1.14 & $-1.31^{*}$ \\
\hline & $(-0.58,1.58)$ & $(0.27,2.78)$ & $(-2.47,0.30)$ & $(-2.25,0.61)$ & $(-0.30,2.59)$ & $(-2.34,-0.27)$ \\
\hline \multirow[t]{2}{*}{$40-54$} & 0.40 & 1.30 & -0.05 & 0.46 & -0.26 & -0.41 \\
\hline & $(-0.44,1.24)$ & $(-0.09,2.70)$ & $(1.66,1.56)$ & $(-0.90,1.82)$ & $(-1.61,1.09)$ & $(-1.68,0.85)$ \\
\hline$>54$ & Ref & Ref & Ref & Ref & Ref & Ref \\
\hline \multicolumn{7}{|l|}{ Gender } \\
\hline \multirow[t]{2}{*}{ Male } & -0.57 & -0.02 & -0.91 & -0.64 & -0.61 & $-1.19^{* *}$ \\
\hline & $(-1.20,0.06)$ & $(-0.83,0.80)$ & $(-1.88,0.06)$ & $(-1.67,0.40)$ & $(-1.35,0.14)$ & $(-1.95,-0.43)$ \\
\hline Female & Ref & Ref & Ref & Ref & Ref & Ref \\
\hline \multicolumn{7}{|c|}{ Urbanization degree } \\
\hline \multirow[t]{2}{*}{ Urban } & $1.66^{*}$ & -1.13 & -0.51 & -0.73 & 0.11 & 0.97 \\
\hline & $(0.16,3.17)$ & $(-3.57,1.32)$ & $(-3.14,2.13)$ & $(-2.13,0.67)$ & $(-1.95,2.17)$ & $(-0.93,2.88)$ \\
\hline \multirow[t]{2}{*}{ Semi-urban } & 0.23 & -0.25 & 0.46 & 0.05 & -0.310 & 0.34 \\
\hline & $(-1.08-1.54)$ & $(-2.06,1.55)$ & $(-2.11,3.03)$ & $(-1.71,1.81)$ & $(-2.26,1.64)$ & $(-1.68,2.36)$ \\
\hline Rural & Ref & Ref & Ref & Ref & Ref & Ref \\
\hline \multicolumn{7}{|l|}{ Education } \\
\hline \multirow[t]{2}{*}{ Low } & -1.17 & -1.05 & -0.38 & -0.88 & -1.45 & $-1.97^{*}$ \\
\hline & $(-2.90,0.57)$ & $(-2.53,0.42)$ & $(2.46,1.70)$ & $(-2.63,0.88)$ & $(-4.27,1.36)$ & $(-3.91,-0.03)$ \\
\hline \multirow[t]{2}{*}{ Medium } & -1.19 & -0.30 & 0.38 & -0.31 & -1.36 & 0.71 \\
\hline & $(-3.16,0.79)$ & $(-1.58,0.98)$ & $(-1.59,2.34)$ & $(-1.82,1.20)$ & $(-4.26,1.52)$ & $(-0.87,2.29)$ \\
\hline High & Ref & Ref & Ref & Ref & Ref & Ref \\
\hline \multicolumn{7}{|l|}{ Income } \\
\hline \multirow[t]{2}{*}{ Low } & -0.05 & 0.94 & 0.74 & $1.68^{*}$ & 0.87 & -0.01 \\
\hline & $(-1.28,1.18)$ & $(-0.50,2.39)$ & $(-1.04,2.52)$ & $(0.18,3.18)$ & $(-0.76,2.50)$ & $(-1.88,1.87)$ \\
\hline \multirow[t]{2}{*}{ Medium } & -0.05 & 1.29 & 1.41 & 0.86 & 1.22 & -0.17 \\
\hline & $(-0.94,0.84)$ & $(-0.07,2.66)$ & $(-0.28,3.10)$ & $(-0.27,2.00)$ & $(-0.21,2.66)$ & $(-1.50,1.17)$ \\
\hline High & Ref & Ref & Ref & Ref & Ref & Ref \\
\hline \multicolumn{7}{|c|}{ Cigarettes/day } \\
\hline \multirow[t]{2}{*}{$1-10$} & $2.90^{* *}$ & $1.83^{* *}$ & 1.80 & $2.97^{*}$ & $4.27^{* *}$ & $2.91^{* *}$ \\
\hline & $(1.14,4.65)$ & $(0.49,3.18)$ & $(-0.16,3.75)$ & $(0.03,5.92)$ & $(1.78,6.75)$ & $(1.19,4.63)$ \\
\hline \multirow[t]{2}{*}{$11-20$} & 1.46 & 0.93 & 0.95 & 1.01 & 2.28 & $2.76^{* *}$ \\
\hline & $(-0.05,2.97)$ & $(-0.18,2.04)$ & $(-0.88,2.79)$ & $(-1.67,3.70)$ & $(-0.27,4.58)$ & $(1.17,4.35)$ \\
\hline \multirow[t]{2}{*}{$21-30$} & 0.92 & 0.24 & 0.32 & -0.39 & 1.26 & 1.49 \\
\hline & $(-0.70,2.55)$ & $(-1.47,1.94)$ & $(-1.77,2.40)$ & $(-3.25,2.49)$ & $(-1.89,4.43)$ & $(-0.63,3.60)$ \\
\hline$>30$ & Ref & Ref & Ref & Ref & Ref & Ref \\
\hline
\end{tabular}

Missing cases were excluded from the analysis. Ref: indicates the reference group in the linear regression analyses. ${ }^{*} p<0.05 ;{ }^{* *} p<0.01 ;{ }^{* * *} p<0.001$.

Relationship between the level of knowledge and labels impact index

Table 7 shows that in all countries, except for Poland, a higher LII score was positively associated with a higher KHR index score, even after controlling for sociodemographic variables and number of cigarettes smoked per day. Higher LII scores were also associated with greater knowledge of specific smoking-related diseases and conditions. 
Table 7. Relationship between knowledge of health effects (KHR index as well as specific knowledge about several health risks) and label impact index (LII)

\begin{tabular}{|c|c|c|c|c|c|c|}
\hline \multirow[b]{2}{*}{ Variable } & Germany & Greece & Spain & Romania & Itungary & Poland \\
\hline & \multicolumn{6}{|c|}{$B\left(95^{\circ} \% C I\right)^{a}$} \\
\hline \multirow[t]{2}{*}{ KHR Index } & $0.33^{* * *}$ & $0.15^{* *}$ & $0.29^{* * *}$ & $0.30^{* * *}$ & $0.30^{* * *}$ & 0.14 \\
\hline & $(0.18,0.49)$ & $(0.04,0.25)$ & $(0.16,0.42)$ & $(0.20,0.40)$ & $(0.13,0.46)$ & $(-0.03,0.30)$ \\
\hline Health Ellect & \multicolumn{6}{|c|}{ Adjusted OR $\left(95^{\circ} \circ \mathrm{CI}\right)^{b}$} \\
\hline \multicolumn{7}{|l|}{ Active smoking } \\
\hline \multirow[t]{2}{*}{ Heart disease } & $1.10^{* * *}$ & 1.02 & $1.16^{* * *}$ & $1.16^{* * *}$ & $1.05^{*}$ & 0.99 \\
\hline & $(1.04,1.15)$ & $(0.96,1.09)$ & $(1.07,1.26)$ & $(1.08,1.23)$ & $(1.01,1.11)$ & $(0.93,1.07)$ \\
\hline \multirow[t]{2}{*}{ Impotence } & $1.08^{* *}$ & $1.05^{*}$ & $1.08^{* *}$ & $1.07^{* * *}$ & $1.06^{* *}$ & $1.06^{* *}$ \\
\hline & $(1.03,1.12)$ & $(1.01,1.09)$ & $(1.02,1.14)$ & $(1.04,1.10)$ & $(1.02,1.10)$ & $(1.01,1.11)$ \\
\hline \multirow[t]{2}{*}{ Lung cancer } & $1.10^{*}$ & $1.21^{*}$ & 1.07 & 1.08 & 1.00 & 1.00 \\
\hline & $(1.01,1.18)$ & $(1.03,1.41)$ & $(0.97,1.18)$ & $(1.00,1.17)$ & $(0.92,1.05)$ & $(0.93,1.05)$ \\
\hline \multirow[t]{2}{*}{ Blindness } & $1.09^{* * *}$ & 1.02 & $1.07^{* * *}$ & $1.07^{* * *}$ & $1.07^{* *}$ & $1.08^{* * *}$ \\
\hline & $(1.04,1.14)$ & $(0.99,1.06)$ & $(1.04,1.12)$ & $(1.04,1.11)$ & $(1.02,1.11)$ & $(1.05,1.11)$ \\
\hline \multirow[t]{2}{*}{ Mouth cancer } & $1.08^{*}$ & 1.05 & $1.14^{* *}$ & $1.10^{* * *}$ & 1.02 & 1.02 \\
\hline & $(1.02,1.14)$ & $(1.00,1.11)$ & $(1.06,1.23)$ & $(1.05,1.16)$ & $(0.98,1.07)$ & $(0.97,1.08)$ \\
\hline \multirow[t]{2}{*}{ Throat cancer } & $1.11^{* *}$ & 1.05 & $1.08^{* *}$ & $1.09^{* *}$ & 1.03 & 0.99 \\
\hline & $(1.05,1.18)$ & $(1.00,1.13)$ & $(1.02,1.14)$ & $(1.03,1.16)$ & $(0.98,1.08)$ & $(0.95,1.05)$ \\
\hline \multirow[t]{2}{*}{ Stroke } & $1.06^{*}$ & 1.04 & $1.06^{* *}$ & $1.11^{* * *}$ & $1.05^{*}$ & 1.02 \\
\hline & $(1.01,1.11)$ & $(0.99,1.09)$ & $(1.01,1.10)$ & $(1.07,1.15)$ & $(1.01,1.09)$ & $(0.97,1.06)$ \\
\hline \multirow[t]{2}{*}{ Emphysema } & 1.05 & 0.97 & 1.03 & $1.07^{* * *}$ & $1.05^{*}$ & 1.00 \\
\hline & $(0.99,1.09)$ & $(0.91,1.02)$ & $(0.98,1.09)$ & $(1.04,1.11)$ & $(1.01,1.09)$ & $(0.95,1.05)$ \\
\hline \multirow[t]{2}{*}{ Bronchitis } & 1.03 & 0.99 & 1.07 & $1.11^{* * *}$ & 1.04 & 1.05 \\
\hline & $(0.97,1.09)$ & $(0.95,1.05)$ & $(0.98,1.19)$ & $(1.06,1.16)$ & $(0.99,1.09)$ & $(1.00,1.10)$ \\
\hline \multicolumn{7}{|l|}{ Secondhand smoke } \\
\hline \multirow[t]{2}{*}{ Lung cancer } & $1.06^{*}$ & 1.04 & $1.07^{* *}$ & $1.05^{*}$ & $1.05^{*}$ & 1.04 \\
\hline & $(1.02,1.11)$ & $(0.99-1.09)$ & $(1.01,1.12)$ & $(1.01,1.09)$ & $(1.01,1.09)$ & $(1.00,1.09)$ \\
\hline \multirow[t]{2}{*}{ Heart attacks } & $1.07^{* *}$ & 1.04 & $1.06^{*}$ & $1.07^{* * *}$ & $1.06^{* *}$ & $1.06^{* *}$ \\
\hline & $(1.03,1.12)$ & $(0.99,1.08)$ & $(1.01,1.11)$ & $(1.04,1.12)$ & $(1.02,1.10)$ & $(1.01,1.10)$ \\
\hline \multirow[t]{2}{*}{ Asthma in children } & $1.08^{* *}$ & $1.05^{*}$ & $1.07^{*}$ & $1.10^{* * *}$ & 1.03 & 1.03 \\
\hline & $(1.03,1.13)$ & $(1.10,1.11)$ & $(1.02,1.13)$ & $(1.06,1.14)$ & $(0.99,1.07)$ & $(0.99,1.08)$ \\
\hline
\end{tabular}

Missing cases were excluded from the analysis. a Linear regression analysis adjusted for age, gender, urbanization degree, income, education level, and cigarettes smoked/day. $b$ Multiple logistic regression analysis adjusted for age, gender, urbanization degree, income, education level, and cigarettes smoked/day. ${ }^{*} p<0.05$, ${ }^{* *} p<0.01,{ }^{* *} p<0.001$.

\section{DISCUSSION}

\section{Principal findings}

The findings of this study indicate that there are significant gaps in smokers' understanding of the health risks of smoking across the six EU MS of the EUREST-PLUS ITC Survey. The vast majority of smokers were aware that smoking causes illnesses such as heart disease, lung and throat cancers. However, more than $20 \%$ of the smokers did not know that tobacco use causes mouth cancer, pulmonary diseases, and stroke. Smokers' knowledge of the causal relationship between smoking and impotence and blindness was significantly lower. Importantly, with the exception of Romania, modest levels of knowledge of the health harms caused by exposure to secondhand smoke were observed across all six EU MS. These findings are similar to that of previous ITC reports for other countries ${ }^{4,19-21}$. All countries in the present study included health warning labels that address risk of heart attack, stroke, lung cancer, and impotence, and to a lesser extent messages regarding blindness, throat, or mouth cancers. Warning labels included non-specific or general messages in terms of lung disease but did not include specific messages related to emphysema or bronchitis. For the most part, warning labels did not include specific messages about 
health effects of secondhand smoke on heart disease and lung cancer or asthma in children. In general, levels of knowledge observed in countries for specific diseases and conditions corresponded to the specific warning labels' messages in circulation.

There were several country-level differences documented, with Romania and Greece having a greater percentage of smokers who recognized the smoking health harms for most of the diseases/ conditions assessed and higher scores on the KHR index. The lowest KHR index scores were documented in Germany and Hungary. These trends did not necessarily correspond to strong health warning labels in these countries in 2016. Given that there are other sources of information on the health risks of smoking, including educational programs in schools, mass media, and medical advice, the higher rates of KHR scores documented in some countries are potentially the consequence of several activities, in addition to warning labels, however this requires further validation ${ }^{22-24}$. For instance, EUREST-PLUS Project findings show that there is an association between high exposure to mass media anti-smoking campaigns and increased knowledge of smoking health harms ${ }^{25}$. Moreover, in Hungary, despite the fact that pictorial warnings were used on tobacco packages, many smokers were using roll-your-own (RYO) tobacco, and packaging of RYO rolling papers and filters, which had no health warning information prior to August $2016^{26}$.

Several sociodemographic factors were associated with KHR index scores although trends varied between countries sampled. In three countries (Germany, Hungary, Poland) women had higher KHR scores, while in Romania and Greece, older age was associated with better KHR scores.

Our study found that only about one-third of smokers noticed warning labels often or very often, and only $20 \%$ read them often or very often. Approximately $5 \%$ of smokers declared that the warning labels made them think 'a lot' about health risks, or made them more likely to quit, and $15 \%$ reported warning labels made them refrain from smoking at least once. Seeing quitline and website information was reported at significantly lower rates in Greece, which is expected given this information did not appear on the warning labels in Greece in 2016 due to the non-existence of a National quitline.
Additionally, there was variability between countries with regard to health warnings' impact on perceptions and actions of the smokers. For instance, Romania and Poland were the countries with the highest LII scores, while Germany and Hungary had the lowest scores. One potential explanation of the low LII score observed in Hungary could be that almost half of smokers in Hungary reported using roll-your-own (RYO) tobacco, while packaging of RYO rolling papers and filters had no health warning information prior to August $2016^{26}$. At the same time, the impact might vary between countries also as a consequence of type (only text or text and pictorials) and dimension of the health warning, and the type of messages ${ }^{2-9}$.

There was a correlation between higher daily cigarette consumption and lower LII scores. Other socioeconomic characteristics also influenced the LII at the country level. In Poland and Romania, the LII was higher among older age groups, while in Greece a reverse situation was observed. In Germany, urban residence was positively associated with a higher LII, while in Poland, the LII was higher among smokers with higher educational level. Other country-level factors, including the prevalence of tobacco use, characteristics of tobacco users and the extent to which comprehensive tobacco policies have been implemented are also expected to play a role in warning label effectiveness. As has been reported by others, it is possible that cultural factors, including receptivity to regulation, may play a role in explaining part of the differences observed between countries ${ }^{27,28}$.

In almost all countries, a significant positive relationship was documented between a KHR and LII scores. Romania and Germany being the countries where this relationship was evident for 10 or 11 of the 12 investigated health risks, while in the other countries the LII scores were associated with the level of knowledge for at least three health risks.

\section{Implications to practice}

These data provide evidence to support the need for stronger education and policies that can enhance the effectiveness of health warnings among EU MS. Previous ITC studies showed that health warnings on cigarette packages are an important source of information regarding health effects of smoking ${ }^{8,10}$. A recent systematic review showed that stronger warnings increased attention to warnings, recall of 
warnings, and thinking about the health risks of smoking, as well as perceptions that warnings reduce smoking and motivate quitting ${ }^{27}$. There is substantial evidence to suggest that strengthening health warnings, such as those outlined in the EU TPD, will increase the effectiveness of warning labels and address some of the gaps in knowledge and salience, as well as promote quitting-related behaviors ${ }^{2,3,5,27}$. The documented gaps in knowledge regarding the health effects of secondhand smoke may also be an important target for public awareness in countries that are still struggling with implementation of comprehensive smoke-free public spaces, cars, and homes.

Importantly, this study serves as a baseline for examining the impact of the TPD, which began its implementation in most countries examined in the EUREST-PLUS ITC Wave 1 Survey in 2016. Future longitudinal studies should investigate changes in knowledge regarding health risks of smoking and the effectiveness of warning labels over time and as a consequence of implementing the TPD in the EU, which will be done within the context of this Horizon 2020 project.

\section{Limitations and strengths}

While this study has many strengths with a large sample size of smokers across six nationally representative EU MS, findings should be interpreted in light of study limitations. First, the study used a cross-sectional design, which allows for the exploration of association rather than causality. The study included only current smokers and as such does not reflect individuals who recently quit smoking. The results are also based on participant self-report that may be subject to respondent and measurement bias. The present study examined variables prior to the implementation of the EU TPD and in some countries large pictorial warnings had been introduced just prior to our assessment and may not be sufficient to measure the effects of warning label changes in these countries. Likewise, KHR may have been influenced by other sources of education including mass media, which were not examined as part of this study. Given that the above warnings differed across countries including the size and health effects featured, some country level differences would be expected that were not controlled for in the analysis. In addition, there are other individual-level factors (e.g. nicotine addiction, presence of smoking-related illness or mental health illness) that might influence outcomes of interest that were not examined as part of this study.

Nevertheless, this study is the only one of its kind to provide a comprehensive baseline of the impact of health warning labels on smokers' knowledge and smoking behaviors across multiple EU MS prior to the full implementation of the EU TPD, which will then be used to evaluate the impact of health warning provisions in a post-TPD assessment.

\section{CONCLUSIONS}

This study has documented important gaps in EU smokers' knowledge of the health effects of active and secondhand smoke and the impact of health warning labels prior to implementation of the EU TPD. These data provide evidence to support the need for stronger education and policies that can enhance the effectiveness of health warnings among EU MS, and will serve as a baseline for examining changes in these indicators following implementation of the TPD.

\section{*EUREST-PLUS consortium members:}

European Network on Smoking and Tobacco Prevention (ENSP), Belgium: Constantine I. Vardavas, Andrea Glahn, Christina N. Kyriakos, Dominick Nguyen, Katerina Nikitara, Cornel Radu-Loghin, Polina Starchenko

University of Crete (UoC), Greece: Aristidis Tsatsakis, Charis Girvalaki, Chryssi Igoumenaki, Sophia Papadakis, Aikaterini Papathanasaki,

Manolis Tzatzarakis, Alexander I. Vardavas

Kantar Public (TNS), Belgium: Nicolas Bécuwe, Lavinia Deaconu, Sophie Goudet, Christopher Hanley, Oscar Rivière

Smoking or Health Hungarian Foundation (SHHF), Hungary: Tibor Demjén, Judit Kiss, Piroska A. Kovacs

Catalan Institut of Oncology (ICO); Bellvitge Biomedical Research Institute (IDIBELL), Spain: Esteve Fernández, Yolanda Castellano, Marcela Fu, Sarah 0. Nogueira, Olena Tigova

Kings College London (KCL), United Kingdom: Ann McNeill, Katherine East, Sara C. Hitchman

Cancer Prevention Unit and WHO Collaborating Centre for Tobacco Control, German Cancer Research Center (DKFZ), Germany: Ute Mons, Sarah Kahnert

National and Kapodistrian University of Athens (UoA), Greece: Yannis Tountas, Panagiotis Behrakis, Filippos T. Filippidis, Christina Gratziou, Paraskevi Katsaounou, Theodosia Peleki, Ioanna Petroulia, Chara Tzavara Aer Pur Romania, Romania: Antigona C. Trofor, Marius Eremia, Lucia Lotrean, Florin Mihaltan

European Respiratory Society (ERS), Switzerland; Goethe University Frankfurt, Germany: Gernot Rohde, Tamaki Asano, Claudia Cichon, Amy Far, Céline Genton, Melanie Jessner, Linnea Hedman, Christer Janson, Ann Lindberg, Beth Maguire, Sofia Ravara, Valérie Vaccaro, Brian Ward

Maastricht University, the Netherlands: Marc Willemsen, Hein de Vries, Karin Hummel, Gera E. Nagelhout

Health Promotion Foundation (HPF), Poland: Witold A. Zatoński, Aleksandra Herbeć, Kinga Janik-Koncewicz, Krzysztof Przewoźniak, Mateusz Zatoński University of Waterloo (UW); Ontario Institute for Cancer Research, Canada: Geoffrey T. Fong, Thomas K. Agar, Pete Driezen, Shannon Gravely, Anne C. K. Quah, Mary E. Thompson 


\section{REFERENCES}

1. World Health Organization. WHO Framework Convention on Tobacco Control. Geneva, Switzerland: WHO Document Production Services; 2003. http://apps.who. int/iris/bitstream/handle/10665/42811/9241591013. pdf;jsessionid=411E B3E4835E57656B2DDFA86D 517D52? sequence=1. Accessed May, 2018.

2. World Health Organization. Evidence Brief. How large pictorial health warning on the packing of tobacco products affects knowledge and behavior. Geneva: WHO; 2014. http://www.euro.who.int/_data/assets/ pdf_file/0006/276558/How-large-pictorial-healthwarnings,-Evidence-Brief-Eng.pdf. Accessed May, 2018.

3. Fong GT, Hammond D, Hitchman SC. The impact of pictures on the effectiveness of tobacco warnings. Bulletin of the World Health Organization. 2009;87:640-643. doi:10.2471/BLT.09.069575

4. Hammond D, Fong GT, McNeill A, Borland R, Cummings KM. Effectiveness of cigarette warning labels in informing smokers about the risks of smoking: findings from the International Tobacco Control (ITC) four country survey. Tob Control. 2006;15(Suppl 3):iii19-iii25. doi:10.1136/tc.2005.012294

5. Monárrez-Espino J, Liu B, Greiner F, Bremberg S, Galanti R. Systematic review of the effect of pictorial warnings on cigarette packages in smoking behavior. Am J Public Health. 2014;104(10):e11-30. doi:10.2105/AJPH.2014.302129

6. Fathelrahman AI, Li L, Borland R, et al. Stronger pack warnings predict quitting more than weaker ones: finding from the ITC Malaysia and Thailand surveys. Tob Induc Dis. 2013;11(September). doi:10.1186/1617-9625-11-20.

7. Borland R, Yong HH, Wilson $\mathrm{N}$, et al. How reactions to cigarette packet health warnings influence quitting: Findings from the ITC four-country survey. Addiction. 2009;104:669-675. doi:10.1111/j.1360-0443.2009.02508.x

8. Borland R, Wilson N, Fong GT, et al. Impact of graphic and text warnings on cigarette packs: findings from four countries over five years. Tobacco Control. 2009;18:358364. doi:10.1136/tc.2008.028043

9. Francis DB, Hall MG, Noar SM, Ribisl KM, Brewer NT. Systematic review of measures used in pictorial cigarette pack warning experiments. Nicotine \& Tobacco Research. 2017;19(10):1127-1137. doi:org/10.1093/ntr/ntx082

10. Directive 2014/40/EU of the European Parliament and of the Council of 3 April 2014 on the approximation of the laws, regulations and administrative provisions of the Member States concerning the manufacture, presentation and sale of tobacco and related products and repealing. Directive 2001/37/EC. OJ 2014 L 127/1. https:// ec.europa.eu/health/sites/health/files/tobacco/docs/ dir_201440_en.pdf. Accessed May, 2018.

11. Commission Implementing Decision (EU) 2015/1842 of 9 October 2015 on the technical specifications for the layout, design and shape of the combined health warnings for tobacco products for smoking. http://eur-lex.europa.eu/legal-content/EN/TXT/ $\mathrm{PDF} /$ ?uri=CELEX:32015D1842\&from=EN. Accessed May, 2018.

12. European Commission. Product Regulation: Tobacco. Health warnings. https://ec.europa.eu/health/tobacco/ law/pictorial_en. Accessed May, 2018.

13. Vardavas CI, Bécuwe N, Demjén T, et al. Study Protocol of European Regulatory Science on Tobacco (EUREST-PLUS): Policy implementation to reduce lung disease. Tob Induc Dis. 2018;16(Suppl 2:A2). doi:10.18332/tid/93305

14. Fong GT, Thompson ME, Boudreau C, et al. The Conceptual Model and Methods of Wave 1 (2016) of the EUREST-PLUS ITC 6 European Countries Survey. Tob Induc Dis. 2018;16(Suppl 2:A3). doi:10.18332/tid/99881

15. Hitchman S, Mons U, Nagelhout GE, et al. Effectiveness of the European Union text-only cigarette health warnings: findings from four countries. Eur J Public Health. 2012;22(5):693-699. doi:10.1093/eurpub/ckr099

16. Green AC, Kaai SC, Fong GT, Drizen P, Quah ACK, Burhoo P. Investigating the effectiveness of pictorial health warnings in Mauritius: Findings from the ITC Mauritius survey. Nicotine Tob Res. 2014;16(9):12401247. doi:10.1093/ntr/ntu062

17. International Agency for Research on Cancer. Methods for Evaluating Tobacco Control Policies. Vol 12. Lyon, France: International Agency for Research on Cancer; 2008:287-317. http://publications.iarc.fr/Book-AndReport-Series/Iarc-Handbooks-Of-Cancer-Prevention/ Methods-For-Evaluating-Tobacco-Control-Policies-2008. Accessed May, 2018.

18. ITG Project. Health warnings on tobacco packages: ITC cross-country comparison report. Ontario, Canada: University of Waterloo; 2012. http://www.itcproject.org/ files/ITC_Cross-Country_Report_-_Warning_LabelsFinal.pdf. Accessed May, 2018.

19. Yang J, Hammond D, Pete Driezen P, Fong GT, Jiang Y. Health knowledge and perception of risks among Chinese smokers and non-smokers: findings from the wave 1 ITC China survey. Tob Control. 2010;19(Suppl 2):i18-i23. doi:10.1136/tc.2009.029710

20. Siahpush M, McNeill A, Hammond D, Fong GT. Socioeconomic and country variations in knowledge of health risks of tobacco smoking and toxic constituents of smoke: results from the 2002 International Tobacco Control (ITC) Four Country Survey Tob Control. 2006;15(Suppl 3):iii65-iii70. doi:10.1136/tc.2005.013276

21. Reid JL, Mutti-Packer S, Gupta PG, et al. Influence of health warnings on beliefs about the health effects of cigarette smoking, in the context of an experimental study in four asian countries. Int J Environ Res Public Health. 2017;14(8). doi:10.3390/ijerph14080868

22. World Health Organization. European Tobacco Control Status Report 2014. Copenhagen, Denmark: World Health 
Organization Regional Office for Europe; 2014. http:// www.euro.who.int/_data/assets/pdf_file/0009/248418/ European-Tobacco-Control-Status-Report-2014-Eng.pdf. Accessed May, 2018.

23. Trofor A, Man M, Marginean C, Filipeanu D, Trofor L. Smoking cessation for free: outcomes of a study in three Romanian clinics. Open Medicine. 2016;11(1):605-610. doi:10.1515/med-2016-0098

24. Lotrean LM, Ailoaiei R, Popa M, de Vries H. Process evaluation of the first computer tailored program for smoking cessation among Romanian smokers. Asian Pac J Cancer Prev. 2014;15(20):8809-8814. doi:10.7314/apjcp.2014.15.20.8809

25. Nogueira SO, McNeill A, Fu M, et al. Impact of anti-smoking advertising on health-risk knowledge and quit attempts across 6 European countries from the EUREST-PLUS ITC Europe Survey. Tob Induc Dis. 2018;16(Suppl 2:A5). doi:10.18332/tid/96251

26. Demjén T, Kiss J, Kovács $P$, et al. The purchase sources of and price paid for cigarettes in six European countries: Findings from the EUREST-PLUS ITC Europe Surveys. Tob Induc Dis. In press.

27. Noar SM, Francis DB, Bridges C, et al. The impact of strengthening cigarette pack warnings: Systematic review of longitudinal observational studies. Soc Sci Med. 2016;164:118-129. doi:10.1177/1077699016674188

28. Nagelhout GE, Willemsen MC, de Vries H, et al. Educational differences in the impact of pictorial cigarette warning labels on smokers: findings from the International Tobacco Control (ITC) Europe surveys. Tobacco Control. 2015;25. doi:10.1136/tobaccocontrol-2014-051971
CONFLICTS OF INTEREST

The authors declare that they have no competing interests, financial or otherwise, related to the current work. L. M. Lotrean reports grants from Aer Pur Romania, during the conduct of the study. K. Przewoźniak reports grants and personal fees from the Polish League Against Cancer, outside the submitted work. C. I. Vardavas reports that he is the Strategic Development Editor of TID and that there are no conflicts of interest with this current work. The rest of the authors have also completed and submitted an ICMJE form for disclosure of potential conflicts of interest.

\section{FUNDING}

The EUREST-PLUS project has received funding from the European Union's Horizon 2020 research and innovation programme under grant agreement No 681109 (CIV) and the University of Waterloo (GTF). Additional support was provided to the University of Waterloo by the Canadian Institutes of Health Research (FDN-148477). GTF was supported by a Senior Investigator Grant from the Ontario Institute for Cancer Research. EF and SON are supported by the Ministry of Universities and Research, Government of Catalonia (2017SGR319). SON received funding from the European Union's 2020 research and innovation programme under the Marie Sklodowska-Curie grant agreement No. 713673 through "La Caixa" INPHINIT Fellowship Grant for Doctoral studies at Spanish Research Centres of Excellence". EF was supported by the Instituto de Salud Carlos III, Government of Spain (INT16/00211 and INT17/00103), co-funded by the European Regional Development Fund (FEDER).

PROVENANCE AND PEER REVIEW

Commissioned; externally peer reviewed. 